\title{
AN EMPIRICAL METHOD OF GROUPING GENOTYPES BASED ON A LINEAR FUNCTION OF THE GENOTYPE-ENVIRONMENT INTERACTION
}

\author{
CHUANG-SHENG LIN and BRIAN THOMPSON \\ Statistical Research Service, Research Branch, Agriculture Canada, Ottawa
}

Received 22.vii.74

\begin{abstract}
SUMMARY
The regression approach for analysing genotype-environmental interaction is extended to include the grouping of genotypes. An unweighted pair-group cluster analysis was applied to a special dissimilarity index, derived from the test statistic for differences among regressions. The resulting groups reflect the general pattern of response to the various environments. The data of Yates and Cochran (1938) were used to illustrate the clustering process.
\end{abstract}

\section{INTRODUCTION}

SincE Tukey (1949) developed a method of using a single degree of freedom to test for the presence of non-additivity in two-way classification data, a number of models have been proposed for interpreting the internal structure of any interaction (see Freeman, 1973, for a literature review). Among these models, two seem to have particular importance for agronomists, breeders and geneticists : the linear model (Mandel, 1961) using a regression approach, and the multiplicative model (Gollob, 1968; Mandel, 1971), using a principal component approach. Application of the principal component approach in the study of genotype-environmental (GE) interactions, which is relatively new, is a powerful tool for analysing interaction especially in situations where models are not easily defined. However, one disadvantage of this approach is that the components are mathematical artifacts without any obvious direct relationship to environmental conditions. Unless these artifacts can be replaced by highly correlated environmental factors, the method is useful only for identifying genotypes with similar interactions but cannot provide information about the relationships of genotypes to environments. Thus, when genotypes suitable for certain specific conditions are sought and when the linear model holds, the regression approach should prove advantageous. The regression approach, originally described by Yates and Cochran (1938), was practically unused until Finlay and Wilkinson (1963) applied it in the study of barley adaptation, but has since emerged as one of the most important tools for handling GE interactions. However, this approach has also frequently been subject to controversy because the marginal means of the environments are used as independent variables in the regression analysis. Freeman and Perkins (1971) have criticised the use of these non-independent environmental measures, claiming that their use violates the fundamental statistical assumptions of regression analysis. Although Freeman (1973) has since withdrawn this criticism, his earlier arguments are frequently used to condemn the approach. Since the purpose of this paper is to propose an extension of the regression approach in order 
to enable the scientist to examine the structure of the interaction more readily, a brief examination of the historical and statistical background will be presented.

When Yates and Cochran (1938) first used the regression approach, i.e. the regression of genotypes on environmental means, their objective was not point estimation but rather the presentation of empirical comparisons among responses of different genotypes. Though a regression technique was used, their method closely resembled separating single degrees of freedom for linear contrasts. Mandel (1961), perhaps unaware of the work of Yates and Cochran, independently developed a similar technique using parallel reasoning. Although Mandel's work was entirely within the context of the $m \times n$ two-way table, his results can be paraphrased as follows. He proposed removing from the interaction sum of squares, a component with $m-1$ degrees of freedom ( $m$ being the number of genotypes) representing the sum of squares for heterogeneity of $m$ regression slopes. He proved that if the slopes are identical for all genotypes, this component is distributed as $\chi^{2}$ and is independent of environmental effects. Thus, if the model is appropriate, the partitioning provides a method of testing for systematic GE interaction.

The problem then is what, if anything, can be done if Mandel's analysis indicates a GE interaction. Yates and Cochran found that by subdividing the five varieties they studied into two groups and calculating the regression for the pooled data, most of the interaction sum of squares could be attributed to the differences between the two regression slopes. This practical result suggests an extension of Mandel's scheme to provide a means of examining the response pattern. When the number of genotypes is small, as in the problem of Yates and Cochran, a satisfactory solution might be attainable by inspecting the individual regression lines. However, in general, a numerical procedure is more desirable.

In this paper, we propose such a procedure which combines the concept of testing for a common regression and the technique of cluster analysis. The data from the paper of Yates and Cochran are used as an example.

\section{Description OF THE MEthod}

Let $Y_{i j}$ represent the observed yield of the $i$ th genotype in the $j$ th environment. Further, let $X_{j}=\bar{Y}_{. j}-\bar{Y}$... . If, in the model

$$
Y_{i j}=\mu+\rho_{i}+\gamma_{j}+\tau_{i j}+\varepsilon_{i j}
$$

the GE interaction $\tau_{i j}$ can be expressed as a linear function of environmental effects, that is, $\beta_{i} \gamma_{j}$, then $Y_{i j}$ can be written as

$$
Y_{i j}=\alpha_{i}+\beta_{i} X_{j}+\Delta_{i j} \text { for } \begin{aligned}
i & =1, \ldots, m \\
j & =1, \ldots, n
\end{aligned}
$$

where $\beta_{i}=1+\beta_{i}^{\prime}$, and $\Delta_{i j}$ is the deviation from the linear regression on $X_{j}$.

It may be noted that if the variance of $\varepsilon_{i j}$ is assumed homogeneous, the variance of the $\Delta_{i j}$ will also be homogeneous within genotypes but will be heterogeneous among genotypes unless the $\beta_{i}$ are equal. Note also that when the yield of each genotype is regressed on the value of $X_{j}$ defined above, 
the intercept of the resulting equation is the mean yield of the genotype and that

$$
\sum_{i=1}^{m} \beta_{i}=m
$$

Because of the second relationship, the grouping of genotypes must be regarded as conditional on the choice of test genotypes.

The object is to group the genotypes with similar intercepts and regression coefficients. To do so a clustering method will be used. The following dissimilarity index is proposed.

Let $S S\left(V_{1} V_{2} \ldots\right)$ represent the sums of squares of deviation from the common regression line for genotypes $V_{1}, V_{2} \ldots$ Then, for example, $S S\left(V_{i}\right)$ simply represents the sum of squares of deviations from the regression line for genotype $V_{i}$. The dissimilarity index for a subset of $r$ genotypes $V_{1}, V_{2} \ldots, V_{r}$ will be defined by

$$
d\left(V_{1} V_{2} \ldots V_{r}\right)=\frac{S S\left(V_{1} V_{2} \ldots V_{r}\right)-\sum_{i=1}^{r} S S\left(V_{i}\right)}{2(r-1) s^{2}}
$$

where

$$
s^{2}=\sum_{i=1}^{m} S S\left(V_{i}\right) /[(m-1)(n-2)] .
$$

The statistic $d(\quad)$ is simply the variance ratio for testing the hypothesis of a common regression line against the alternative hypothesis of $r$ independent regression lines. Note that the degrees of freedom used in determining $s^{2}$ are $(m-1)(n-2)$ instead of $m(n-2)$ owing to the use of the environmental means as independent variables (see Mandel, 1961). A small value of $d(\quad)$ indicates similar regression lines; larger values indicate increasingly dissimilar ones. It can be shown (see Appendix 1) that

$$
d\left(V_{1} V_{2} \ldots V_{r}\right)=\frac{2}{r(r-1)} \sum_{i^{\prime}>i} d\left(V_{i} V_{i^{\prime}}\right)
$$

that is, the dissimilarity index for $r$ genotypes is equal to the mean of the indices for all $\left(\begin{array}{l}r \\ 2\end{array}\right)$ possible pairs of genotypes. Thus this index conforms to the conditions set by Sokal and Michener (1958) in describing the unweighted pair-group cluster method, and hence their algorithm can be applied. It should be noted that the dissimilarity index is not a distance measure since the triangular inequality does not hold in general. However, the sequence of the smallest dissimilarity indices chosen in the $m-1$ cycles of the clustering process is monotonically non-decreasing (see Appendix 2), i.e.

$$
d_{1}^{\prime}() \leqq d_{2}^{\prime}() \leqq \ldots \leqq d_{m-1}^{\prime}(),
$$

where $d_{i}^{\prime}($ ) represents the smallest index in the $i$ th clustering cycle.

A difficulty of this and other clustering processes is to define a suitable stopping criterion. The authors have found it useful to take as an empirical criterion the $F$ test for a common regression line, stopping the process when

$34 / 2-B$ 
the smallest dissimilarity index exceeds the $F$ value for $r$ and $(m-1)(n-2)$ degrees of freedom at a predetermined probability level.

\section{Numerical Example}

This example is taken from the paper of Yates and Cochran (1938). The two-way table of total yields for five barley varieties at six locations is given in table 1 .

TABLE 1

Two-way table of variety by location (based on the total of three replications and two years' data), cited from the paper by Yates and Cochran (1938)

\begin{tabular}{lcccccccc}
\multicolumn{10}{c}{ Variety } & Coded & \multicolumn{1}{c}{ Location } \\
\cline { 3 - 8 } & & & 2 & 3 & 4 & 5 & 6 & Mean \\
Manchusia & $(1)$ & $161 \cdot 7$ & $247 \cdot 0$ & $185 \cdot 4$ & $218 \cdot 7$ & $165 \cdot 3$ & $154 \cdot 6$ & $188 \cdot 78$ \\
Svansota & $(2)$ & $187 \cdot 7$ & $257 \cdot 5$ & $182 \cdot 4$ & $183 \cdot 3$ & $138 \cdot 9$ & $143 \cdot 8$ & $182 \cdot 27$ \\
Velvet & $(3)$ & $200 \cdot 1$ & $262 \cdot 9$ & $194 \cdot 9$ & $220 \cdot 2$ & $165 \cdot 8$ & $146 \cdot 3$ & $198 \cdot 37$ \\
Trebi & $(4)$ & $196 \cdot 9$ & $339 \cdot 2$ & $271 \cdot 2$ & $266 \cdot 3$ & $151 \cdot 2$ & $193 \cdot 6$ & $236 \cdot 40$ \\
Peatland & $(5)$ & $182 \cdot 5$ & $253 \cdot 8$ & $219 \cdot 2$ & $200 \cdot 5$ & $184 \cdot 4$ & $190 \cdot 1$ & $209 \cdot 08$ \\
Mean & & $185 \cdot 78$ & $272 \cdot 08$ & $210 \cdot 62$ & $217 \cdot 80$ & $161 \cdot 12$ & $165 \cdot 68$ & $202 \cdot 18$
\end{tabular}

The values of $X_{j}$ as defined in the previous section were $-16 \cdot 4,69 \cdot 9$, $8.44,15.62,-41.06$ and -36.5 for locations 1 to 6 respectively. For each variety in turn, the yields were regressed against these values; the resulting regression analyses are shown in table 2. The pooled error estimate is

TABLE 2

Regression analyses for five varieties

$\begin{array}{crccc}\text { Coded Varieties } & \begin{array}{c}\text { SS due to } \\ \text { regression }\end{array} & \begin{array}{c}\text { SS of deviations } \\ \text { from regression }\end{array} & \begin{array}{c}\text { Intercept } \\ \left(\alpha_{i}\right)\end{array} & \begin{array}{c}\text { slope } \\ \left(\beta_{i}\right)\end{array} \\ 1 & 6046.55 & 702.55 & 188 \cdot 78 & 0.8840 \\ 2 & 8256.52 & 794.49 & 182 \cdot 27 & 0.9862 \\ 3 & 7599.89 & 827.90 & 198.37 & 0.9462 \\ 4 & 21965.73 & 1358.29 & 236.40 & 1.6086 \\ 5 & 3209.31 & 546.60 & 205.08 & 0.6149\end{array}$

$s^{2}=4229 \cdot 83 / 16=264 \cdot 36$ and the dissimilarity matrix for the first cycle is as follows

$\begin{array}{ccccc}\text { coded varieties } & 2 & 3 & 4 & 5 \\ 1 & 0.4034 & 0.6050 & 17.5585 & 1.9289 \\ 2 & & 1.4836 & 19 \cdot 7370 & 4 \cdot 0610 \\ 3 & & & 11.7302 & 1 \cdot 1372 \\ 4 & & & & 13.4922\end{array}$


The unweighted pair-group cluster method gives

\begin{tabular}{clccc} 
& & \multicolumn{3}{c}{ number of varieties } \\
clustering cycle $(i)$ & varieties grouped & $d^{\prime}{ }_{i}(\quad)$ & in the group $(r)$ & $F(r, 16)$ \\
1 & $(12)$ & $0 \cdot 4034$ & 2 & $3 \cdot 63$ \\
2 & $(12), 3$ & $0 \cdot 8307$ & 3 & $3 \cdot 24$ \\
3 & $(123), 5$ & $1 \cdot 6032$ & 4 & $3 \cdot 01$ \\
4 & $(1235), 4$ & $7 \cdot 2137$ & 5 & $2 \cdot 85$
\end{tabular}

where the $F$ values refer to the 5 per cent level of probability. At the fourth cycle, the dissimilarity index, far exceeds the $F$ value. It would appear then that the data suggest two distinct groupings: variety 4 alone, and the other four varieties. This is consistent with the conclusions drawn by Yates and Cochran.

To illustrate the effectiveness of grouping, the analyses of variance based on Mandel's procedure (1961) and on the two groupings are shown in table 3. Most of the variation among varieties is included in the betweengroup component. Similarly, most of the variation among slopes is accounted for by the slope $\times$ group interaction. Both results reflect a satisfactory grouping.

TABLE 3

Analyses of variance based on Mandel and the newly proposed grouping schemes

\begin{tabular}{|c|c|c|c|}
\hline \multicolumn{4}{|c|}{ Mandel } \\
\hline Source & d.f. & SS & MS \\
\hline Locations (L) & 5 & $42441 \cdot 82$ & $8488 \cdot 36$ \\
\hline Varieties (V) & 4 & 10618.95 & 2654.99 \\
\hline$V \times L$ & 20 & $8866 \cdot 02$ & - \\
\hline Slopes & 4 & $4636 \cdot 18$ & $1159 \cdot 18$ \\
\hline Residuals & 16 & $4229 \cdot 84$ & $264 \cdot 37$ \\
\hline
\end{tabular}

\begin{tabular}{|c|c|c|c|}
\hline \multicolumn{4}{|c|}{ Groups } \\
\hline Source & d.f. & SS & MS \\
\hline Location (L) & 5 & $42441 \cdot 82$ & $8488 \cdot 36$ \\
\hline Groups (G) & 1 & $8782 \cdot 57$ & $8782 \cdot 57$ \\
\hline Varieties/G & 3 & $1837 \cdot 38$ & $612 \cdot 46$ \\
\hline $\mathrm{G} \times \mathrm{L}$ & 5 & $5628 \cdot 51$ & - \\
\hline Slope $\times G$ & 1 & $3930 \cdot 65$ & $3930 \cdot 65$ \\
\hline Dev. $\times G$ & 4 & $1697 \cdot 86$ & $424 \cdot 46$ \\
\hline $\mathrm{V} \times \mathrm{L} / \mathrm{G}$ & 15 & $3237 \cdot 51$ & - \\
\hline Slope $\times$ V/G & 3 & 705.53 & $235 \cdot 18$ \\
\hline Dev. $\times \mathrm{V} / \mathrm{G}$ & 12 & $2531 \cdot 98$ & $211 \cdot 00$ \\
\hline
\end{tabular}

\section{Discussion}

The objective of the regression approach in the study of GE interaction is to separate systematic interaction from random variation. If the response of each genotype across environments can be approximated by a straight line, the non-parallelism of response will be indicated by the difference among slopes. The present grouping procedure is an attempt to extend the regression approach by systematically grouping genotypes into homogeneous subsets within which all genotypes share a common regression (i.e. no interaction and no difference between means).

An empirical solution is sought using a cluster analysis in which the test statistics for differences among regression lines is used as a measure of dissimilarity among regression lines. If the dissimilarity index of two genotypes is defined by equation (1) for all pairs of combinations, and if Sokal and Michener's (1958) unweighted pair-group method is applied to this $m \times m$ dissimilarity matrix, then the new indices constructed in each clustering cycle will be the test statistics for differences among regression lines for the 
corresponding genotypes. Thus, the grouping based on the critical $F$-value as a stopping criterion should avoid heterogeneity of regression lines within groups and, therefore in the resulting ANOVA the mean squares for withingroup sources of variation should be approximately homogeneous.

The grouping method simplifies the interpretation of the data by achieving the following:

(i) The number of regression lines for genotypes is reduced to the number of groups. This makes it easier for scientists to identify the general response pattern.

(ii) The genotypes in the same group have approximately the same response to environments. If there is a well-established genotype in a group, the other genotypes included in the group should be suitable for the same environments where the established genotype is grown.

(iii) The grouping leads to subdivision of the sum of squares in Mandel's analysis of variance to more useful components. The variation attributable to group characteristics can be isolated both for the difference among genotype means and from the difference among slopes.

The grouping need not be based jointly on the slopes and intercept of linear regression. For example, it is possible to group only on the basis of slope and it is also possible to group if the model is a non-linear polynomial. In such cases, the formula for the dissimilarity index given in (1) can be replaced by the appropriate test statistics. Although the proposed grouping method was developed as a numerical solution for identifying systematic response pattern among GE interactions, this method can be applied to a general situation in which the objective is to subdivide a set of regression equations (all equation having the same set of independent variables) into homogeneous subsets.

The example included in this paper has been chosen for its simplicity and for its familiarity in the present context. However, the authors have applied the method to larger data sets involving as many as 25 varieties. In every case, the procedure lead to relatively few groups and, as in the results of table 3 , the slope $\times$ group interaction accounted for almost all of the GE interaction.

Acknowledgements.-The authors wish to express their appreciation to their colleagues and in particular to Mr L. P. Lefkovitch and Mrs P. Morse, for their useful criticisms and suggestions during the course of this work.

\section{RefERENCES}

FINLAY, K. W., AND WILKINSON, G. M. 1963. The analysis of adaptation in plant-breeding program. Aust. F. Agr. Res., 14, 742-754.

Freeman, G. H. 1973. Statistical methods for the analysis of genotype-environmental interactions. Heredity, 31, 339-354.

FREEMAN, G. H., AND PERKINS, J. M. 1971. Environmental and genotype-environmental components of variability. VII. Relations between genotypes grown in different environments and measures of these environments. Heredity, 27, 15-23.

GоLLOB, H. F. 1968. A statistical model which combines features of factor analytic and analysis of variance techniques. Psychometrika, 33, 73-116.

MANDEL, J. 1961. Non-additivity in two-way analysis of variance. F. Am. Statist. Ass., $56,878-888$.

MANDEL, J. 1971. A new analysis of variance model for non-additive data. Technometrics, 13, $1-18$. 
SOKAL, R. R., AND MICHENER, C. D. 1958. A statistical model for evaluating systematic relationships. Univ. Kansas Sci. Bull., 38, 1409-1438.

TUKEY, J. W. 1949. One degree of freedom for non-additivity. Biometrics, 5, 232-242.

yates, F., and cochran, w. G. 1938. The analysis of groups of experiments. F. Agri. Sci., $28,556-580$.

\section{Appendix 1}

Lemma 1. For $r>1$,

$$
d\left(V_{1} V_{2} \ldots V_{r}\right)=\frac{2}{r(r-1)}\left[\sum_{i^{\prime}>i} d\left(V_{i} V_{i^{\prime}}\right)\right] .
$$

Proof. Let $Y_{i j}$ represent the observed yield of the $i$ th genotype in the $j$ th environment and $X_{j}$ represent the environmental effect of the $j$ th environment defined as $X_{j}=\bar{Y}_{. j}-\bar{Y}$..; then $\hat{Y}_{i j}$ can be defined as a linear function of $X_{j}$ as follows:

where

$$
\begin{aligned}
\widehat{Y}_{i j}=\bar{Y}_{i .}+b_{i} X_{j}, \text { for } \quad i & =1, \ldots, m \\
j & =1, \ldots, n,
\end{aligned}
$$

$$
\sum_{i=1}^{m} b_{i}=m .
$$

We have the following equations.

$$
\begin{aligned}
S S\left(V_{i}\right) & =\sum_{j=1}^{n}\left(Y_{i j}-\bar{Y}_{i .}-b_{i} X_{j}\right)^{2} \\
& =\sum_{j}\left(Y_{i j}-\bar{Y}_{i .}\right)^{2}-b_{i}^{2} \sum_{j} X_{j}^{2}, \\
S S\left(V_{i} V_{i^{\prime}}\right) & =\sum_{j}\left(Y_{i j}-\bar{Y}_{i .}\right)^{2}+\sum_{j}\left(Y_{i^{\prime} j}-\bar{Y}_{i^{\prime}} .\right)^{2}+\frac{1}{2} n\left(\bar{Y}_{i .}-\bar{Y}_{i^{\prime}} .\right)^{2} \\
& -\frac{1}{2}\left(b_{i}+b_{i^{\prime}}\right)^{2} \sum_{j} X_{j}^{2},
\end{aligned}
$$

and

$$
\begin{aligned}
S S\left(V_{1} V_{2} \ldots V_{r}\right)=\sum_{i=1}^{r} \sum_{j}\left(Y_{i j}-\bar{Y}_{i .}\right)^{2}+n \sum_{i}\left(\bar{Y}_{i .}-\sum_{i=1}^{r} \bar{Y}_{i .} / r\right)^{2} & \\
& -\frac{1}{r}\left(\sum_{i} b_{i}\right)^{2} \sum_{j} X_{j}^{2}
\end{aligned}
$$

By definition

$$
\begin{aligned}
2(r-1) s^{2} d\left(V_{1} V_{2} \ldots V_{r}\right)= & S S\left(V_{1} V_{2} \ldots V_{r}\right)-\sum_{i=1}^{r} S S\left(V_{i}\right) \\
= & -\frac{1}{r}\left(\sum_{i} b_{i}\right)^{2} \sum_{j} X_{j}^{2}+n \sum_{i}\left(\bar{Y}_{i .}-\sum_{i=1}^{r} \bar{Y}_{i .} / r\right)^{2} \\
& +\sum_{i} b_{i}^{2} \sum_{j} X_{j}^{2} \\
= & \left\{\sum_{j} X_{j}^{2}\left[\left(b_{1}-b_{2}\right)^{2}+\left(b_{1}-b_{3}\right)^{2}+\ldots+\left(b_{r-1}-b_{r}\right)^{2}\right]\right. \\
& \left.+n\left[\left(\bar{Y}_{1}-\bar{Y}_{2}\right)^{2}+\left(\bar{Y}_{1}-\bar{Y}_{3 .}\right)^{2}+\ldots+\left(\bar{Y}_{r-1}-\bar{Y}_{r .}\right)^{2}\right]\right\} / r .
\end{aligned}
$$


Since

$$
\begin{aligned}
d\left(V_{i} V_{i^{\prime}}\right) & =\left[S S\left(V_{i} V_{i^{\prime}}\right)-S S\left(V_{i}\right)-S S\left(V_{i^{\prime}}\right)\right] / 2 s^{2} \\
& =\frac{1}{4 s^{2}}\left[\sum_{j} X_{j}^{2}\left(b_{i}-b_{i^{\prime}}\right)^{2}+n\left(\bar{Y}_{i .}-\bar{Y}_{i^{\prime}}\right)^{2}\right] . \\
\therefore d\left(V_{1} V_{2} \ldots V_{r}\right) & =\frac{2}{r(r-1)}\left[\sum_{i^{\prime}>i} d\left(V_{i} V_{i^{\prime}}\right)\right] .
\end{aligned}
$$

\section{Appendix 2}

LEMMA 2.

$$
d_{2}^{\prime}() \leqq d_{2}^{\prime}() \leqq \ldots \leqq d_{i}^{\prime}() \leqq \ldots \leqq d_{m-1}^{\prime}() .
$$

Proof. To show the above relationship, we shall consider two cases: (i) a single genotype is to be added to the group; (ii) more than one genotype is to be added. The proofs of these two cases will be given for specific examples. However, the technique used to prove these two cases can be extended to the proof of the more general case.

Case 1. Suppose $d_{1}^{\prime}\left(V_{1} V_{2}\right)$ is the smallest index in the first clustering cycle, we want to show that

$$
d_{1}^{\prime}\left(V_{1} V_{2}\right) \leqq d\left(V_{1} V_{2} V_{r}\right) \text { for } r \neq 1,2
$$

and that $d_{1}^{\prime}\left(V_{1} V_{2}\right) \leqq d_{2}^{\prime}()$ in general.

From lemma 1,

$$
d\left(V_{1} V_{2} V_{r}\right)=\frac{1}{3}\left[d_{1}^{\prime}\left(V_{1} V_{2}\right)+d\left(V_{1} V_{r}\right)+d\left(V_{2} V_{r}\right)\right]
$$

Subtracting $d_{1}^{\prime}\left(V_{1} V_{2}\right)$ from both sides, we have

$$
d\left(V_{1} V_{2} V_{r}\right)-d_{1}^{\prime}\left(V_{1} V_{2}\right)=\frac{1}{3}\left\{\left[d\left(V_{1} V_{r}\right)-d_{1}^{\prime}\left(V_{1} V_{2}\right)\right]+\left[d\left(V_{2} V_{r}\right)-d_{1}^{\prime}\left(V_{1} V_{2}\right)\right]\right\} .
$$

By definition $d_{1}^{\prime}\left(V_{1} V_{2}\right)$ is the smallest index in the lst cycle, which implies

$$
\begin{aligned}
& d_{1}^{\prime}\left(V_{1} V_{2}\right) \leqq d\left(V_{1} V_{r}\right), \\
& d_{1}^{\prime}\left(V_{1} V_{2}\right) \leqq d\left(V_{2} V_{r}\right), \\
\therefore \quad & d\left(V_{1} V_{2} V_{r}\right)-d_{1}^{\prime}\left(V_{1} V_{2}\right) \geqq 0 .
\end{aligned}
$$

Since there are only two types of index in the 2 nd cycle, i.e.

and

$$
d\left(V_{i} V_{j}\right) \text { for } i, j \neq 1,2,
$$

$$
\begin{aligned}
& d_{2}\left(V_{1} V_{2} V_{r}\right) \text { for } r \neq 1,2 . \\
& \therefore \quad d_{1}^{\prime}\left(V_{1} V_{2}\right) \leqq \\
& d_{2}^{\prime}() .
\end{aligned}
$$

Case 2. Suppose $d_{1}^{\prime}\left(V_{1} V_{2}\right)$ is the smallest index in the lst cycle, and $d_{2}^{\prime}\left(V_{3} V_{4}\right)$ is the smallest index in the 2 nd cycle, we want to show that $d_{2}^{\prime}\left(V_{3} V_{4}\right) \leqq d\left(V_{1} V_{2} V_{3} V_{4}\right)$, and that $d_{2}^{\prime}\left(V_{3} V_{4}\right) \leqq d_{3}^{\prime}()$ in general. 
From lemma 1,

$$
\begin{aligned}
d\left(V_{1} V_{2} V_{3} V_{4}\right)= & \frac{1}{6}\left[d_{1}^{\prime}\left(V_{1} V_{2}\right)+d\left(V_{1} V_{3}\right)+d\left(V_{1} V_{4}\right)+d\left(V_{2} V_{3}\right)+d\left(V_{2} V_{4}\right)\right. \\
& \left.+d\left(V_{3} V_{4}\right)\right] \\
= & \frac{1}{2}\left[d\left(V_{1} V_{2} V_{3}\right)+d\left(V_{1} V_{2} V_{4}\right)\right]+\frac{1}{6}\left[d\left(V_{3} V_{4}\right)-d_{1}^{\prime}\left(V_{1} V_{2}\right)\right] .
\end{aligned}
$$

Subtracting $d_{2}^{\prime}\left(V_{3} V_{4}\right)$ from both sides, we have

$$
\begin{aligned}
d\left(V_{1} V_{2} V_{3} V_{4}\right)-d_{2}^{\prime}\left(V_{3} V_{4}\right)=\frac{1}{2}\left\{\left[d\left(V_{1} V_{2} V_{3}\right)-d_{2}^{\prime}\left(V_{3} V_{4}\right)+\right.\right. & {\left.\left[d\left(V_{1} V_{2} V_{4}\right)-d_{2}^{\prime}\left(V_{3} V_{4}\right)\right]\right\} } \\
+ & +\frac{1}{6}\left[d\left(V_{3} V_{4}\right)-d_{1}^{\prime}\left(V_{1} V_{2}\right)\right] .
\end{aligned}
$$

Since $d_{2}^{\prime}\left(V_{3} V_{4}\right)$ is the smallest index in 2 nd cycle,

and by definition

$$
\begin{aligned}
& d_{2}^{\prime}\left(V_{3} V_{4}\right) \leqq d\left(V_{1} V_{2} V_{3}\right), \\
& d_{2}^{\prime}\left(V_{3} V_{4}\right) \leqq d\left(V_{1} V_{2} V_{4}\right), \\
& d_{1}^{\prime}\left(V_{1} V_{2}\right) \leqq d_{2}^{\prime}\left(V_{3} V_{4}\right), \\
\therefore \quad & d_{3}\left(V_{1} V_{2} V_{3} V_{4}\right)-d_{2}^{\prime}\left(V_{3} V_{4}\right) \geqq 0 .
\end{aligned}
$$

Since there are only four types of indices in the 3 rd cycle, i.e.

We know that

$$
\begin{aligned}
& \left.\begin{array}{l}
d_{3}\left(V_{i} V_{j}\right) \text { for } i, j \neq 1,2,3,4, \\
d_{3}\left(V_{1} V_{2} V_{r}\right) \\
d_{3}\left(V_{3} V_{4} V_{r}\right)
\end{array}\right\} \text { for } r \neq 1,2,3,4, \\
& d_{3}\left(V_{1} V_{2} V_{3} V_{4}\right) .
\end{aligned}
$$

and

$$
d\left(V_{i} V_{j}\right) \geqq d_{2}^{\prime}\left(V_{3} V_{4}\right),
$$

$$
d\left(V_{1} V_{2} V_{r}\right) \geqq d_{2}^{\prime}\left(V_{3} V_{4}\right),
$$

by definition of $d_{2}^{\prime}()$ and from case 1

$$
\begin{aligned}
& d\left(V_{3} V_{4} V_{r}\right) \geqq d_{2}^{\prime}\left(V_{3} V_{4}\right), \\
\therefore \quad & d_{2}^{\prime}\left(V_{3} V_{4}\right) \leqq d_{3}^{\prime}() .
\end{aligned}
$$

